University of Nebraska - Lincoln

DigitalCommons@University of Nebraska - Lincoln

2015

Intrinsic magnetic properties of L10 FeNi obtained from meteorite NWA 6259

\author{
Eric Poirier \\ MEDA Engineering and Technical Services \\ Frederick E. Pinkerton \\ GM R\&D Center, frederick.e.pinkerton@gm.com \\ Robert Kubic \\ GM R\&D Center \\ Raja K. Mishra \\ GM R\&D Center \\ Nina Bordeaux \\ Northeastern University
}

See next page for additional authors

Follow this and additional works at: https://digitalcommons.unl.edu/physicsskomski

Part of the Physics Commons

Poirier, Eric; Pinkerton, Frederick E.; Kubic, Robert; Mishra, Raja K.; Bordeaux, Nina; Mubarok, Arif; Lewis, Laura H.; Goldstein, Joseph I.; Skomski, Ralph; and Barmak, Katayun, "Intrinsic magnetic properties of L10 FeNi obtained from meteorite NWA 6259" (2015). Ralph Skomski Publications. 95.

https://digitalcommons.unl.edu/physicsskomski/95

This Article is brought to you for free and open access by the Research Papers in Physics and Astronomy at DigitalCommons@University of Nebraska - Lincoln. It has been accepted for inclusion in Ralph Skomski Publications by an authorized administrator of DigitalCommons@University of Nebraska - Lincoln. 


\section{Authors}

Eric Poirier, Frederick E. Pinkerton, Robert Kubic, Raja K. Mishra, Nina Bordeaux, Arif Mubarok, Laura H. Lewis, Joseph I. Goldstein, Ralph Skomski, and Katayun Barmak 


\title{
Intrinsic magnetic properties of L1, FeNi obtained from meteorite NWA 6259
}

\author{
Eric Poirier, ${ }^{1, a)}$ Frederick E. Pinkerton, ${ }^{2, b)}$ Robert Kubic, ${ }^{2}$ Raja K. Mishra, ${ }^{2}$ Nina Bordeaux, ${ }^{3}$ \\ Arif Mubarok, ${ }^{4, \mathrm{c})}$ Laura H. Lewis, ${ }^{3}$ Joseph I. Goldstein, ${ }^{4}$ Ralph Skomski, ${ }^{5}$ \\ and Katayun Barmak ${ }^{6}$ \\ ${ }^{1}$ MEDA Engineering and Technical Services, Southfield, Michigan 48075, USA \\ ${ }^{2}$ Chemical Sciences and Materials Systems Lab, GM R\&D Center, Warren, Michigan 48090, USA \\ ${ }^{3}$ Department of Chemical Engineering, Northeastern University, Boston, Massachusetts 02115, USA \\ ${ }^{4}$ Department of Mechanical and Industrial Engineering, University of Massachusetts, Amherst, \\ Massachusetts 01003, USA \\ ${ }^{5}$ Department of Physics and Astronomy, University of Nebraska, Lincoln, Nebraska 68588, USA \\ ${ }^{6}$ Department of Applied Physics and Applied Mathematics, Columbia University, New York, \\ New York 10027, USA
}

(Presented 7 November 2014; received 21 September 2014; accepted 15 November 2014; published online 25 March 2015)

\begin{abstract}
FeNi having the tetragonal $\mathrm{L}_{0}$ crystal structure is a promising new rare-earth-free permanent magnet material. Laboratory synthesis is challenging, however, tetragonal $\mathrm{L}_{0} \mathrm{FeNi}-$ the mineral "tetrataenite" - has been characterized using specimens found in nickel-iron meteorites. Most notably, the meteorite NWA 6259 recovered from Northwest Africa is 95 vol. \% tetrataenite with a composition of 43 at. \% Ni. Hysteresis loops were measured as a function of sample orientation on a specimen cut from NWA 6259 in order to rigorously deduce the intrinsic hard magnetic properties of its $\mathrm{L} 1_{0}$ phase. Electron backscatter diffraction showed that NWA 6259 is strongly textured, containing $\mathrm{L} 1_{0}$ grains oriented along any one of the three equivalent cubic directions of the parent fcc structure. The magnetic structure was modeled as a superposition of the three orthonormal uniaxial variants. By simultaneously fitting first-quadrant magnetization data for 13 different orientations of the sample with respect to the applied field direction, the intrinsic magnetic properties were estimated to be saturation magnetization $4 \pi M_{s}=14.7 \mathrm{kG}$ and anisotropy field $H_{a}=14.4 \mathrm{kOe}$. The anisotropy constant $K=0.84 \mathrm{MJ} / \mathrm{m}^{3}$ is somewhat smaller than the value $K=1.3 \mathrm{MJ} / \mathrm{m}^{3}$ obtained by earlier researchers from nominally equiatomic FeNi prepared by neutron irradiation accompanied by annealing in a magnetic field, suggesting that higher Ni content (fewer Fe antisite defects) may improve the anisotropy. The fit also indicated that NWA 6259 contains one dominant variant ( $62 \%$ by volume), the remainder of the sample being a second variant, and the third variant being absent altogether. (C 2015 AIP Publishing LLC. [http://dx.doi.org/10.1063/1.4916190]
\end{abstract}

Increasing demand for high strength magnets in the rapidly growing applications of vehicle traction motors and wind energy harvesting has kindled new research efforts to develop alternative permanent magnet materials free of strategically limited elements. Since the 1970s and 1980s, the rare-earth "supermagnets" based on Sm-Co and Nd-Fe-B have dominated the high-energy-product magnet market. Combining the high magnetization of $\mathrm{Fe}$ and $\mathrm{Co}$ with the high magnetocrystalline anisotropy energy of the rare earths $\mathrm{Nd}, \mathrm{Pr}, \mathrm{Sm}$, Dy, and $\mathrm{Tb}$, these magnets typically have energy products $(B H)_{\max }$ of $25-50$ MGOe $\left(200-400 \mathrm{~kJ} / \mathrm{m}^{3}\right)$. However, the current and projected future growth in demand engendered by high volume traction motors (about $1 \mathrm{~kg}$ per motor) and wind generators (about $1000 \mathrm{~kg}$ per generator) puts increasing stress on the availability and cost of rare-earth magnets. The rare earths have been identified by the U.S. Department of Energy as highly critical elements for clean energy applications with

\footnotetext{
${ }^{a)}$ Current address: Product Development, Ford Motor Company, Dearborn, Michigan 48121, USA.

b) Author to whom correspondence should be addressed. Electronic mail: frederick.e.pinkerton@gm.com.

${ }^{c}$ Current address: Deringer-Ney, Bloomfield, Connecticut 06002, USA.
}

medium or high supply risk, ${ }^{1}$ prompting research on rareearth-free permanent magnets.

Here, we consider FeNi having the chemically ordered and crystallographically anisotropic $\mathrm{L} 1_{0}$ structure as a potential hard magnet candidate. The tetragonal $\mathrm{L}_{0}$ structure (space group $\mathrm{P} 4 / \mathrm{mmm}$ ) consists of alternating layers of $\mathrm{Fe}$ and $\mathrm{Ni}$ atoms stacked in the direction parallel to the tetragonal c-axis. It is isomorphic to other notable $\mathrm{L} 1_{0}$ magnetic compounds such as chemically ordered FePt, FePd, CoPt, and MnAl. The first reports of the magnetic properties of $\mathrm{L} 1_{0} \mathrm{FeNi}$ were presented more than five decades ago by Paulevé et al., ${ }^{2}$ who synthesized chemically ordered $\mathrm{L}_{0}$ FeNi from the chemically disordered fcc parent phase by fast neutron flux (energy $\mathrm{E}>1 \mathrm{MeV}$ ) bombardment in an applied magnetic field. Irradiation was carried out below the $\mathrm{L} 1_{0}$ to fcc order-disorder transformation temperature, estimated to be $T_{\text {od }}=320-330^{\circ} \mathrm{C}$, in order to form the ordered structure. Subsequent work by Néel et al. ${ }^{3}$ starting from single crystal fcc FeNi deduced that the $\mathrm{L} 1_{0}$ phase formed in three distinct variants, corresponding to chemical ordering along the three equivalent cubic axes of the fcc parent phase. These authors concluded that the neutron bombardment contributed to the kinetics of $\mathrm{L} 1_{0}$ formation by defect creation and atomic displacement at temperatures below 
$320^{\circ} \mathrm{C}$ that would otherwise be insufficient to promote atomic diffusion. Analysis of the magnetization data extracted bulk anisotropy constants $K_{1}=0.32 \mathrm{MJ} / \mathrm{m}^{3}$ and $K_{2}=0.23 \mathrm{MJ} / \mathrm{m}^{3}$ with a saturation magnetization $4 \pi M_{s}=16 \mathrm{kG}$. Refinement of the data by Paulevé et al. ${ }^{4}$ obtained $K_{1}=1.3 \mathrm{MJ} / \mathrm{m}^{3}$ and $K_{2}=-0.1 \mathrm{MJ} / \mathrm{m}^{3}$ for a single variant, with the easy axis perpendicular to the alternating $\left(\begin{array}{lll}0 & 0 & 1\end{array}\right)$ planes of $\mathrm{Fe}$ and $\mathrm{Ni}$ (i.e., the tetragonal $c$-axis). In addition to neutron bombardment, small samples of $\mathrm{L}_{0}$ FeNi have been synthesized by highenergy electron irradiation of electron-transparent foil ${ }^{5}$ and more recently by molecular beam epitaxy of FeNi films showing selected characteristics consistent with the tetragonal compound. $^{6-10}$ Laboratory synthesis of bulk $\mathrm{L1}_{0}$ FeNi remains challenging because its low order-disorder temperature $\left(T_{o d}=320^{\circ} \mathrm{C}\right)$ impedes conversion from the stable high temperature fcc phase by ordinary thermal processes.

Fortunately, we can use naturally occurring $\mathrm{L}_{0} \mathrm{FeNi}$ as a surrogate to understand its potential as a permanent magnet. $\mathrm{L}_{0}$ FeNi has been identified as a component in some classes of meteorites, ${ }^{11,12}$ where it is known as the mineral tetrataenite. The extremely low cooling rates of asteroid metal (as low as $0.3 \mathrm{~K}$ per million years) allow chemical ordering even at the very low diffusion rates that exist below $320^{\circ} \mathrm{C}$. In particular, a Ni-rich meteorite recovered from Northwest Africa, NWA 6259, is comprised of $95 \%$ bulk tetrataenite by volume, the remainder being non-magnetic FeNi phosphides and sulfides. The $\mathrm{L} 1_{0}$ phase in NWA 6259 contains $43 \pm 1.3$ at. $\%$ $\mathrm{Ni}$, near the Fe-rich end of the $\mathrm{L}_{0}$ phase field. ${ }^{13}$ Our previous studies of NWA 6259 have confirmed via transmission electron microscopy (TEM) the presence of $\mathrm{L}_{0}$ FeNi with at least two variants of the $c$-axis orientation, correlated the intrinsic magnetic properties with the microstructure of bulk tetrataenite, ${ }^{14}$ and revealed the extraordinary temperature stability of the magnetic properties. ${ }^{15}$ In the latter paper, hard-direction magnetization data were used to estimate the anisotropy field of the NWA 6259 tetrataenite as $H_{a}=20 \pm 3 \mathrm{kOe}$, most likely toward the lower end of the range $(\sim 17 \mathrm{kOe})$. In this report, we use a much more extensive set of magnetization data taken at $15^{\circ}$ intervals over a $180^{\circ}$ rotation of the sample to extract a substantially more rigorous estimate of $H_{a}$.

A $6 \mathrm{~mm}$ diameter cylinder was cut from a slab section of NWA 6259 with a thickness of approximately $3 \mathrm{~mm}$. Its density was measured pycnometrically to be $8.15 \mathrm{~g} / \mathrm{cm}^{3}$. Mo $\mathrm{k}_{\alpha}$ $\mathrm{X}$-ray diffraction (XRD) and electron back-scatter diffraction (EBSD) were conducted on a polished surface of the cylinder to determine texture and crystallographic orientation, as previously reported. $^{14}$

Hysteresis loops were measured using a LakeShore Model 7410 vibrating sample magnetometer (VSM). The large mass of the cylinder proved problematical for magnetic measurements with the VSM; at applied fields greater than $15 \mathrm{kOe}$, the small magnetic field gradients in the electromagnet caused a deflection of the sample at the end of its mounting rod, creating spurious moment readings. The cylinder was thus cut into a cube approximately $2.6 \mathrm{~mm}$ on a side to permit VSM measurements up to $28 \mathrm{kOe}$ applied field (about $24 \mathrm{kOe}$ internal field $H_{\text {int }}$ ). The measurements were conducted in the plane of the sample, perpendicular to the original cylinder axis, at 13 orientation angles $\theta_{j}$ every $15^{\circ}$ over a $180^{\circ}$ rotation of the sample. The demagnetization correction $D$ used to obtain the internal field $H_{\text {int }}$ was experimentally derived from a magnetically soft pure Ni cube.

The XRD area pattern resolved slightly arced discrete spots, indicating that the specimen was a highly textured polycrystal with minor variations in crystal axis orientation among the crystallites. Quantitative EBSD further elucidated the crystallographic orientation. Although EBSD was not sensitive to the very weak superlattice peaks characteristic of the $\mathrm{L} 1_{0}$ ordering, it proved to be an effective probe of the overlying fcc parent structure. ${ }^{14}$ The Euler angles (Bunge representation) of the fcc structure were determined to be $\varphi_{1}=5.7^{\circ}, \Phi=-31.8^{\circ}$, and $\varphi_{2}=173.3^{\circ}$. It is recognized that in the EBSD measurement the specimen was mounted with a randomly chosen rotational orientation, thus only the reference frame $\mathrm{z}$-axis (surface normal) is unique; the reference $\mathrm{x}$ and $\mathrm{y}$ axes of the measuring system with respect to the in-plane structure are arbitrary. We have made use of this degree of freedom to specifically choose $\varphi_{1}$ such that the Euler angle rotations place the [100] cubic axis in the $\mathrm{X}-\mathrm{Z}$ plane of the reference axis system. The Euler angles then translate to a physical picture in which the [100] direction is canted by $3.5^{\circ}$ out of the plane of the original cylinder face, ${ }^{16}$ and the [001] direction is tilted by $31.8^{\circ}$ relative to the surface normal. The standard deviation of the distribution of polycrystal axes about this average direction is about $2.5^{\circ}$. The cubic axes [100], [010], and [001] are the three directions along which the FeNi planes in different crystallites can order to form the three variants of the $\mathrm{L} 1_{0}$ structure, as shown schematically in Fig. 1. As previously noted, at least two variants have been observed, and in principle all three variants may be present in the sample.

Fig. 2 shows the upper half of the $M(H)$ hysteresis loops for four exemplary measurement angles. To avoid confusion, the other nine measured loops are not shown, as they all fall within the envelope defined by the loops at $202^{\circ}$ and $262^{\circ}$. Note that the extrema of the envelope are separated by $60^{\circ}$, not by $90^{\circ}$ as would be expected for a simple uniaxial system, because the magnetic response is a superposition of the responses from more than one uniaxial variant; the magnetic response cannot be treated as a simple uniaxial system, for which we could simply measure the transverse magnetization. ${ }^{17}$ It is fascinating that the meteorite as recovered, having formed an equilibrium microstructure far from optimized for hard magnetic properties, already shows an intrinsic coercivity $H_{c i}$ of about $1 \mathrm{kOe}$, an observation that bodes well for its promise as a permanent magnet material.

To quantitatively evaluate the saturation magnetization $4 \pi M_{s}$ and the anisotropy field $H_{a}$ of this tetrataenite specimen, we modeled the specimen's magnetic response by superimposing the responses of up to three mutually orthogonal magnetic uniaxial variants with tetragonal $c$-axes along the [100], [010], or [001] directions as given by the Euler angles. For a given $M\left(H, \theta_{j}\right)$ curve at orientation angle $\theta j$ of the applied field (and, equivalently, of the magnetization measurement direction), we calculated the angles $\eta_{i, j}(i=[100],[010],[001])$ between the applied field direction $\hat{H}$ and the three [100], [010], and [001] variant easy axis directions. For each variant, we then calculated the equilibrium canting angle $\alpha_{i, j}\left(H, \theta_{j}\right)$ of the magnetization away from its easy direction point-by-point 

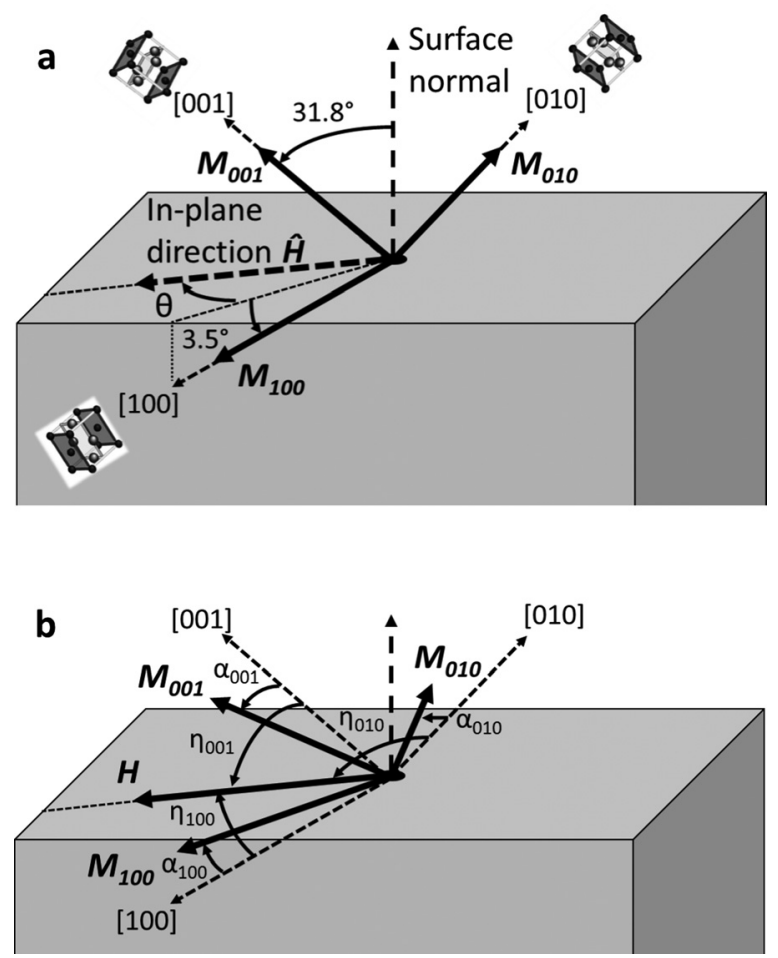

FIG. 1. (a) Schematic depiction of the magnetic structure, consisting of three orthogonal uniaxial $\mathrm{L} 1_{0}$ variants with the [100] variant lying $3.5^{\circ}$ away from parallel to the sample surface, and the [001] variant canted $31.8^{\circ}$ from the surface normal; (b) a field $H$ applied parallel to the sample surface at angle $\theta$ rotates the $M_{s}$ for each variant to a new equilibrium direction in the plane defined by its easy axis and the direction of $H$.

for every experimental $H$ value using a standard coherent rotation model. In this model, $\alpha_{i, j}$ is the angle which minimizes the total energy $E_{t o t}=E_{m}+E_{a}$, where $E_{m}$ is the magnetostatic energy $E_{m}=-H M_{s} \cos \left(\eta_{i, j}-\alpha_{i, j}\right), E_{a}$ is the anisotropy energy $E_{a}=K \sin ^{2} \alpha_{i, j}$, and $K$ is the first order anisotropy energy $K=1 / 2 H_{a} M_{s}$. The magnetization at each $H$ value was then computed as the sum of the components of magnetization along $\hat{H}$ originating from the three variants, $M_{\text {calc }}\left(H, \theta_{j}\right)$ $=\Sigma^{3}{ }_{i=1} f_{i} M_{s} \cos \left(\eta_{i, j}-\alpha_{i, j}\right){ }^{18}$ Each variant was weighted by its

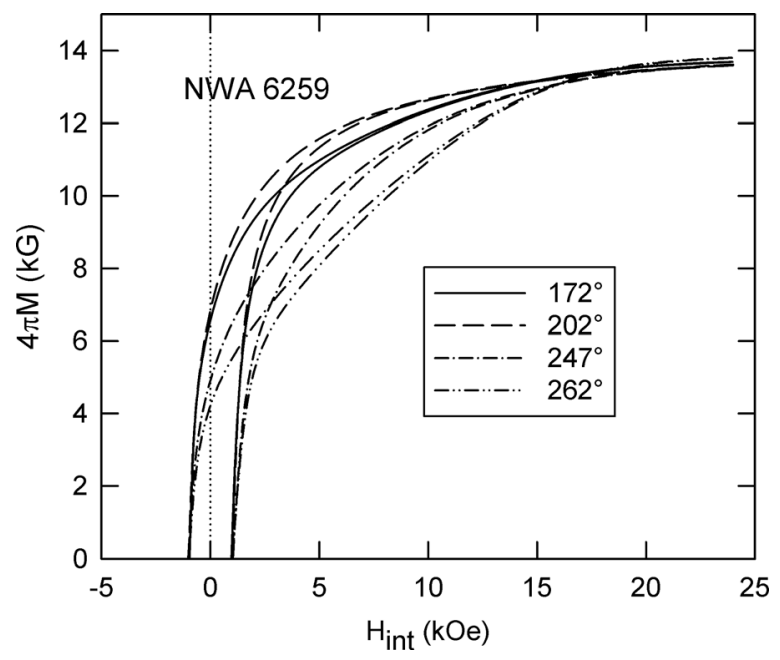

FIG. 2. Hysteresis behavior of NWA 6259 tetrataenite at selected measurement angles. The full set of measured magnetization curves falls within this envelope. volume fraction $f_{i}$, subject to the constraint that $f_{100}+f_{010}+f_{001}=1$, and in general the three variants need not be equally present in the sample. Finally, because the sample was mounted in the magnetometer with unknown rotational orientation, we adopted another parameter $\beta$ to represent this initial misorientation angle between the Euler angle reference frame $\mathrm{x}$-axis (dictated by the [100] crystallite direction) and the zero angle index of the magnetometer. We emphasize that $\beta$ is not a magnetic or microstructural parameter, but rather describes the arbitrary rotational orientation in mounting the specimen in the VSM.

To ensure that the coherent rotation criterion was met, we avoided regions of the hysteresis loop where domain wall motion might contribute to the change in magnetization. We considered data from the first quadrant of the hysteresis loop as the field decreased from the fully magnetized state (upper arm), and only down to a cutoff field $H_{\min }$ below which domain wall formation and motion are likely to impact the $M(H)$ curves. The fits were remarkably insensitive to $H_{\text {min }}$ within the interval $5 \mathrm{kOe}<H_{\min }<11 \mathrm{kOe}$. The fits failed at low $H_{\min }$ as magnetization reversal by domain wall formation and motion became significant, and at high $H_{\min }$ because there was insufficient data at fields below $H_{a}$. For this work, we adopted a conservative value in the middle of the range, $H_{\text {min }}=8 \mathrm{kOe}$.

We then performed a least-squares minimization fit of $M_{\text {calc }}(H)$ to the observed $M(H)$ curves using five fitting parameters: $4 \pi M_{s}, H_{a}, f_{100}, f_{010}$, and $\beta$ (the third volume fraction $\left.f_{001}=1-f_{100}-f_{010}\right)$. We simultaneously fit all 13 $4 \pi M(H)$ curves in the region between the maximum field and $H_{\min }$, approximately $2200 M(H)$ points, using a single set of five parameters. For simplicity, we assumed that the variants were independent and non-interacting, and we did not consider the small dispersion in the easy axis directions.

Fig. 3 shows the best fit of the model for several representative $\mathrm{M}(\mathrm{H})$ curves. We again forego showing all fits due to the substantial overlap of data from different curves. We obtained $\quad 4 \pi M_{s}=14.0 \mathrm{kG}, \quad H_{a}=14.4 \mathrm{kOe}, \quad \beta=82.0^{\circ}$, $f_{100}=0.62, f_{010}=0.38$, and $f_{001}=0$, with a good Coefficient of Determination $R^{2}=0.95$. The value of $\beta$ was used to determine the angle designations in the figures. Fig. 4 further demonstrates the good quality of the fit. Shown are the angular dependence of the observed (solid symbols) and fit (open symbols) magnetizations at $H_{\text {int }}=H_{\min }=8 \mathrm{kOe}$ and at the maximum field $H_{\text {int }}=24 \mathrm{kOe}$. The fit reproduces the observed angular dependence quite well at both field extremes, including the oscillation of magnetization with angle at $H_{\text {int }}=24 \mathrm{kOe}$, although the fit overestimates the size of the variation. The worst fits to individual $M(H)$ curves tend to be those similar to the $82^{\circ}$ fit in Fig. 3, where the fit produces a sharp break in the $4 \pi M(H)$ curve near $H_{a}$. The measured data are smoother through this region, perhaps reflecting the dispersion in the distribution of easy axes that was not included in the model. The fit parameters did not change appreciably if we expanded the fitting range to a more aggressive value of $H_{\min }=5 \mathrm{kOe}$.

The results presented here provide detailed measurement and analysis of the magnetic properties of $\mathrm{L}_{0}$-type $\mathrm{FeNi}$, tetrataenite, derived from the meteorite NWA 6259. 


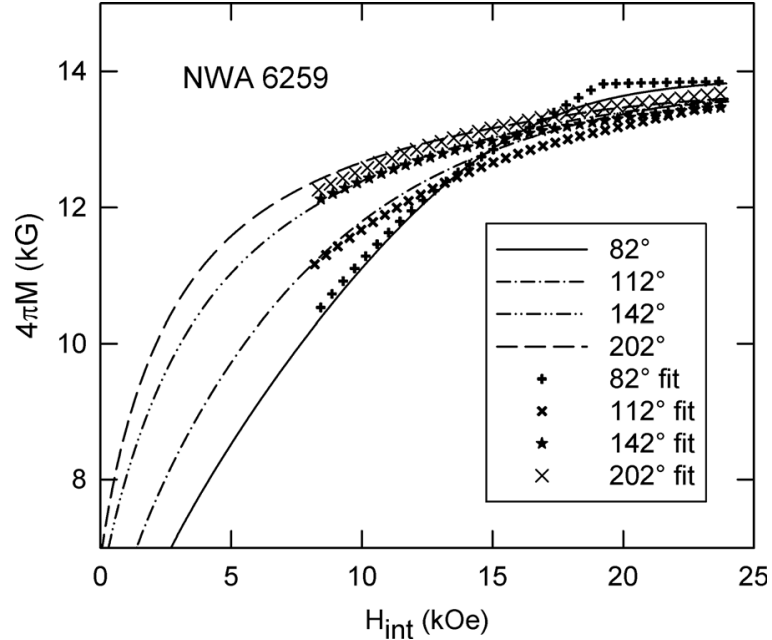

FIG. 3. Selected first quadrant magnetization curves starting from the maximum applied field, along with the model fits as described in the text.

After correcting the measured magnetization for the 95 vol. \% tetrataenite fraction in NWA 6259, the true magnetization of $\mathrm{L}_{0} \mathrm{FeNi}$ at 43 at. $\% \mathrm{Ni}$ is $4 \pi M_{s}=14.7 \mathrm{kG}$, in accord with previous measurements of NWA $6259 .{ }^{14}$ The anisotropy field is modestly smaller than the previous estimate. ${ }^{15}$ Together these data yield an anisotropy constant $K=0.84 \mathrm{MJ} / \mathrm{m}^{3}$. This value falls into the range of $K$ values reported by Kojima et $\mathrm{al}^{7}$ for artificially grown ordered FeNi films of different $\mathrm{Fe} / \mathrm{Ni}$ compositions, but it is somewhat less than the value of $K_{1}=1.3 \mathrm{MJ} / \mathrm{m}^{3}$ obtained by Paulevé in neutron-irradiated bulk FeNi. ${ }^{4}$ The Paulevé result suggests that the $\mathrm{L} 1_{0}$ composition to obtain optimal magnetic properties in bulk FeNi may lie closer to equiatomic FeNi with fewer Fe antisite defects. ${ }^{14}$

While we had anticipated that NWA 6259 would contain all three chemically ordered $\mathrm{L} 1_{0}$-type variants, the fit systematically drove the volume fraction of the [001] variant to zero. Attempts to force non-zero [001] variant volume fraction, for example, by fixing all of the volume fractions at $1 / 3$, invariably produced poor fits (e.g., $R^{2}=0.72$ ). Clearly, this specimen taken from NWA 6259, at least, does not contain all three variants, but rather has one dominant variant comprising about $62 \%$ of the volume, the remainder of the specimen is a second variant, and the third variant is absent altogether. Having a strongly favored variant is important for the extraction of magnetic properties as reported here, but also bodes well for achieving fully oriented, single variant $\mathrm{L} 1_{0} \mathrm{FeNi}$ permanent magnets in the future.

The combination of high saturation magnetization with a reasonably high magnetocrystalline anisotropy holds great promise for developing a high performance magnet based on $\mathrm{L}_{0}$ FeNi. That the NWA 6259 meteorite exhibits significant coercivity $H_{c i}=1 \mathrm{kOe}$, even after low-temperature annealing for cosmological times, graphically illustrates the potential of this rare-earth-free magnet material. For the $43 \% \mathrm{Ni} \mathrm{L1} 1_{0}$ FeNi composition studied here, $H_{a}$ and $4 \pi M_{s}$ are comparable, thus the energy product of an optimized magnet will likely be coercivity-limited $\left(H_{c i}<B_{r} / 2\right)$. Even so, a theoretical energy product of $\sim 40$ MGOe may reasonably be

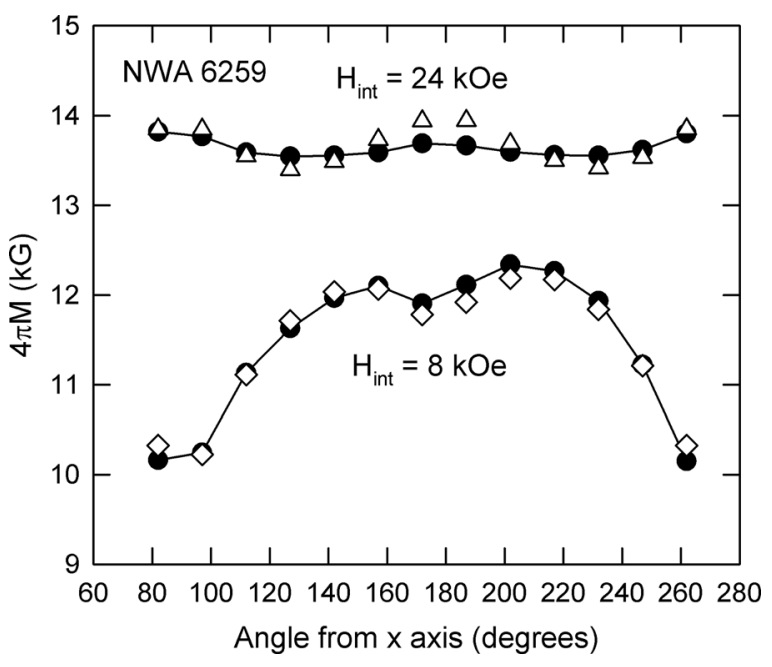

FIG. 4. Angular variation of the first quadrant magnetization measured at internal fields of $24 \mathrm{kOe}$ and $8 \mathrm{kOe}$. Filled circles are the experimental data, and open symbols correspond to the model fit as described in the text.

anticipated. The Paulevé result ${ }^{4}$ suggests that higher $(\mathrm{BH})_{\max }$ may be possible if $H_{a}$ can be increased through compositional modification. Furthermore, in contrast to Nd-Fe-B, the hard magnetic properties of tetrataenite vary only weakly with temperature, ${ }^{15}$ making $\mathrm{L} 1_{0}$ FeNi a very attractive candidate for high temperature applications.

This work was supported by ARPA-E REACT Grant No. 0472-1537.

${ }^{1}$ U.S. Department of Energy, "Critical materials strategy," December 2011. ${ }^{2}$ J. Paulevé, D. Dautreppe, J. Laugier, and L. Néel, C. R. Acad. Sci. 254, 965 (1962).

${ }^{3}$ L. Néel, J. Paulevé, R. Pauthenet, J. Laugier, and D. Dautreppe, J. Appl. Phys. 35, 873 (1964).

${ }^{4}$ J. Paulevé, A. Chamberod, K. Krebs, and A. Bourret, J. Appl. Phys. 39, 989 (1968).

${ }^{5}$ K. B. Reuter, D. B. Williams, and J. I. Goldstein, Metall. Mater. Trans. A 20, 711 (1989).

${ }^{6}$ T. Shima, M. Okamura, S. Mitani, and K. Takanashi, J. Magn. Magn. Mater. 310, 2213 (2007).

${ }^{7}$ T. Kojima, M. Ogiwara, M. Mizuguchi, M. Kotsugi, T. Koganezawa, T. Ohtsuki, T. Y. Tashiro, and K. Takanashi, J. Phys.: Condens. Matter 26, 064207 (2014).

${ }^{8}$ M. Mizuguchi, T. Kojima, M. Kotsugi, T. Koganezawa, K. Osaka, and K. Takanashi, J. Magn. Soc. Jpn. 35, 370 (2011).

${ }^{9}$ M. Mizuguchi, S. Sekiya, and K. Takanashi, J. Appl. Phys. 107, 09A716 (2010).

${ }^{10}$ T. Kojima, M. Mizuguchi, T. Koganezawa, K. Osaka, M. Kotsugi, and K. Takanashi, Jpn. J. Appl. Phys., Part 1 51, 010204 (2012).

${ }^{11}$ J. F. Albertsen, G. B. Jenssen, and J. M. Knudsen, Nature 273, 453 (1978).

${ }^{12}$ J. F. Albertsen, Phys. Scr. 23, 301 (1981).

${ }^{13}$ C. W. Yang, D. B. Williams, and J. I. Goldstein, J. Phase Equilib. 17, 522 (1996).

${ }^{14}$ L. H. Lewis, A. Mubarok, E. Poirier, N. Bordeaux, P. Manchanda, A. Kashyap, R. Skomski, J. I. Goldstein, F. E. Pinkerton, R. K. Mishra, R. C. Kubic, Jr., and K. Barmak, J. Phys.: Condens. Matter 26, 064213 (2014).

${ }^{15}$ L. H. Lewis, F. E. Pinkerton, N. Bordeaux, A. Mubarok, E. Poirier, J. I. Goldstein, R. Skomski, and K. Barmak, IEEE Magn. Lett. 5, 5500104 (2014).

${ }^{16}$ The nearly in-plane alignment of the [100] variant must be regarded as fortuitous.

${ }^{17}$ It is also not equivalent to a system with cubic anisotropy, for which the maximum angle between the magnetization and the nearest easy axis would be $55^{\circ}$. Here, angles nearing $90^{\circ}$ to one of the uniaxial variants are possible.

${ }^{18}$ For an isotropic distribution of uniaxial easy axes, this would correspond to the Stoner-Wohlfarth model. Here, there are three well defined uniaxial directions. 